\title{
DISCUSSION AFTER PAPER BY SUGIMOTO
}

Massevitch to Sugimoto: Could you please give an estimate of how your results for late stages (for $12 M_{\odot}$ and $30 M_{\odot}$ ) will change, if the initial chemical composition is changed?

Sugimoto: I do not think that the results depend very much on composition.

Massevitch: But the depth of the convective envelope would be changed - in what direction?

Sugimoto: I do not think that the change is very great.

Fricke to Sackmann: Did you use a self-consistent method to calculate the joining up of the inner and outer convection zones?

Sackmann: No, we looked at the problem from the other way around. For this project, we have simply assumed that such a link-up of the convection zones does occur and then asked what kind of surface abundances would result. It turned out that we obtained all of the peculiar abundances of the light elements observed in the late-type stars of spectral types, S, C, BaII and CH. We could, in fact, even account for many abundance peculiarities of $\mathrm{R}$ Coronae Borealis stars. Such argument strongly supports our initial assumptions.

However, independent of the project discussed above, we have investigated plausibility arguments for this assumption. We have found (Smith and Sackmann, 1973, submitted to Astrophys. J.) that, from the point of view of stellar evolution, such a link-up would not be impossible.

Schwarzschild to Sugimoto: I very much admire the thorough and beautifully systematic results which you, Dr Sugimoto, have just shown us. I feel quite persuaded that the sequence of evolutionary events as a function of stellar mass which you have presented may qualitatively turn out to be right. But I am somewhat worried that quantitatively these results may still be beset by a rather greater uncertainty than might appear because the range of possible convective efficiency which you have used ( $\alpha=1.0$ to 1.5 ) seems to me substantially smaller than the range of uncertainty in our knowledge regarding convection.

Sugimoto: Of course you are right but we can say something in extreme cases. In some situations no penetration of convection occurs even if infinite mixing length is used.

Arnett to Sugimoto: I am impressed by Sugimoto's results, and am particularly intrigued by his results for massive stars with variable effectiveness of the lepton neutrino losses. If the neutrino losses are too low, mixing of the carbon-oxygen zone will overproduce ${ }^{14} \mathrm{~N}$. I estimate that on the average (20 $M_{\odot}$ star?) one should mix less than $6 \%$ of the $\mathrm{C}$, O region. Can Dr Sugimoto tell me what value of the Universal Fermi interaction that implies? 
Sugimoto: I am not certain of the exact figure but it should be more than $10 \%$ of the standard value.

Ergma to Sugimoto: I should like to point out that the structure and depth of the convection zones in stars with deep convective envelopes depends not only on the assumed value of $\alpha$ but also, as was shown by Henyey, Vardya and Bodenheimer (Astrophys. J. 142, 841, 1965) and more recently by Ergma and Massevitch (Astron. Nachr. 293, 145, 1971) is very sensitive to the values of coefficients $\alpha_{1}, \gamma$ and $v$ which are used for description of convective transfer in the mixing length theory. Taking, for example, $\alpha=1.0$ but varying $\alpha_{1}, \gamma$ and $v$, one can obtain a large variety of depths for convective envelopes. Therefore one has to be very careful in using these results for interpretation of physical processes in convective envelopes. 